\title{
An Automated Inventory Management System
}

\author{
Melancy Mascarenhas \\ Information Technology \\ Shree Rayeshwar Institue of \\ Engineering and Information \\ Technology, Shiroda-Goa \\ Shiroda, India
}

\author{
Anand Lamani \\ Information Technology \\ Shree Rayeshwar Institue of \\ Engineering and Information \\ Technology, Shiroda-Goa \\ Marcel, India
}

\author{
Chandrakant Matkar \\ Information Technology \\ Shree Rayeshwar Institue of \\ Engineering and Information \\ Technology, Shiroda-Goa \\ Ponda, India
}

\author{
Amogh Ramchandra Dessai \\ Information Technology \\ Shree Rayeshwar Institue of Engineering and \\ Information \\ Technology, Shiroda-Goa \\ Marcel, India
}

\author{
Anush Kotharkar \\ Information Technology \\ Shree Rayeshwar Institue of Engineering and \\ Information \\ Technology, Shiroda-Goa \\ Vasco, India
}

\begin{abstract}
The aim of the proposed system is to create a web based system which will contain basic modules for tracking of inventory levels, re-ordering of items, issuing and report generation of refreshment items, organizing inventory data, status alerts, and printing reports. The system analyses the total sale of menu items and proportionately deducts appropriate amount from the resource database. Then it compares the current available resource with the threshold level of each ingredient. If particular ingredients are below the threshold, It generates a purchase order(printable) for those items(s) and sends an email to the manager(admin) for approval.
\end{abstract}

It also generates and display monthly/yearly report in form of statistics and graphs. Apriori clustering algorithm is used to find frequently issued items to various units.

\section{Keywords}

Inventory, Apriori, web

\section{INTRODUCTION}

Inventory management is important to any business as it helps them to prevent stock-outs, manage multiple locations, and ensure accurate record-keeping. An inventory management system makes these processes easier than trying to do them manually. The existing inventory Management system uses Ms Excel to store stock data. However using Excel to track large qualities of items increases the likelihood of data entry errors, especially if your inventory moves frequently from location to location. Also having a single Excel workbook limits user access. Excel lacks real time inventory and also limits user ability to quickly analyse historical data. The proposed system is designed in a way to facilitate insertion, deletion and changes of inventory data directly using designed GUI interface without interacting with the database tables. There are several features within the approach such as statistics, search function, login details and much more.

The system displays brief instructions about using the system which user must follow before entering the login page. An admin logs in using the username and password details stored in the login database table. After successful login, he can access all the functionalities of the system. The system will maintain detailed master list of items along with their threshold reorder levels, normal levels and opening stock data. System will have GUI to modify any of these data. Project will also inco-operate Apriori algorithm to find frequently issued to various units and analysing the items sent to various purposes and narrations.

\section{TECHNOLOGY USED a. $H T M L$}

HTML is a mark-up language used for structuring and presenting content for world wide web. It can be assisted by technologies such as Cascading Style Sheets (CSS) and scripting languages such as JavaScript. The key has been to develop the language with the latest multimedia support while keeping it easy to read and understandable by computers and device(web browsers, parsers, etc.). It contains various text that are predefined and can be used at any time in association with the relative ending tag. HTML elements forms building block of all websites. HTML allows images and objects to be imported and can be used to create functional forms. It can be used to create structured documents by denoting structural semantics for texts, heading, paragraphs, lists, links, quotes and other items.

b. $M y S Q L$

Many of the world's largest and fast growing organization including Facebook, Google and adobe rely on My SQL to save time and money powering their high volume websites, business critical systems and packaged software. It is relational database system user access to a number of databases. MySQL works on many different system platforms, including aixOS, BSDi, free BSD, HP OSX, Microsoft windows, netBSD, Novell NetWare, open BSD, open Solaris, UnixWare.

c. $P H P$

PHP is an acronym for "PHP Hypertext Processor". PHP is a separate server-side language intended for web development but is also used as a standard programming language. PHP is now installed on over 244 million websites and 2.1 million web servers. The PHP code is interpreted by a web server with a PHP processor module, which produces the resulting web page: PHP commands can be installed directly from an HTML source document rather than calling an external file to 
process data.

\section{d. JAVASCRIPT}

JavaScript(JS) is an interpreted computer programming language. The writing language developed by Netscapes to enable Web writers to design functional sites. Although it share many of the features of the full java language, it was developed independently. JavaScript can interact with HTML, source code enabling web authors to spice up their sites with dynamic contents. As part of a web browser, implementation allows client-side scripts to interact with the user, manage the browser, interact with one another, and modify the document content displayed. And it has become the norm in server-side game development and desktop applications.

\section{e. CSS}

Cascading Style Sheet (CSS) is a style sheet language used to describe the appearance and format of document restoration in embedded language. While most often used to change the style of web pages and user interfaces return in HTML and XHTML. The language can be applied to any kind of XML document, including plain XML, XUL and SVG. Along with HTML and Java Script, CSS is a corner Stone technology used by various websites to create visually using web pages, user interface for web applications, user interface for many mobile applications. CSS is designed primarily to enable the separation of documents contents from document presentation, including function such as the layout, colour and font. This classification can provide content access, provide more flexibility and control in the presentation of specification features and enable more HTML pages to share the formatting correctly CSS in a separate. CSS file, reduce complexity and repetition in the structural content, such as semantically insignificant tables that were widely used to format pages before consistent CSS rendering was available in all major browsers.

\section{f. Bootstrap}

Bootstrap is a free and open source CSS application dedicated to responsive, first-rate web development. It has a CSS and JavaScript template used for typing, forms, buttons, navigation and other interfaces.

\section{SYSTEM DESCRIPTION}

A. System Flow

\section{a. Login Page}

The first visible screen before starting the application which will take you to the home page after entering correct username and password(mandatory).

\section{b. Home}

The home page of the web application provides link to every aspect of the web application like Item list, Inventory entry, Month-wise inventory, Register a new admin, current stock status, send an email, etc.

\section{c. Register a new admin}

It displays the registration page for registering a new admin Upon entering in email id, contact number, username, password and confirm password field, the user gets notified in case the input is invalid.

\section{d. Master Item List}

This form is used to create master item list. All the fields like date, item name, opening stock, reorder level, normal level in this forms are mandatory.

\section{e. Inventory Entries}

This form is used to enter inventory details. When issue radio button is selected stock gets deducted from available stocks and when receipt radio button is selected stock gets added to the available stocks.

\section{f. Inventory Details}

This webpage displays the inventory details of every items with its current stock status available.

\section{B. Design Flow}

a. Activity Diagram

Activity diagram is defined as a UML diagram that focuses on the execution and flow of the behaviour of a system instead of implementation. It is also called object-oriented flowchart. Activity diagrams consist of activities that are made up of actions which apply to behavioural modelling technology.

In the below activity diagram, the admin first has to login into system with username and password. The system validates the user, if confirmed he can access the commands like add, modify, delete, search, update, search and view report. The system can also get access to all the commands.

To register a new admin, he must be a super admin. Super admin must login into the account and he register a new admin.

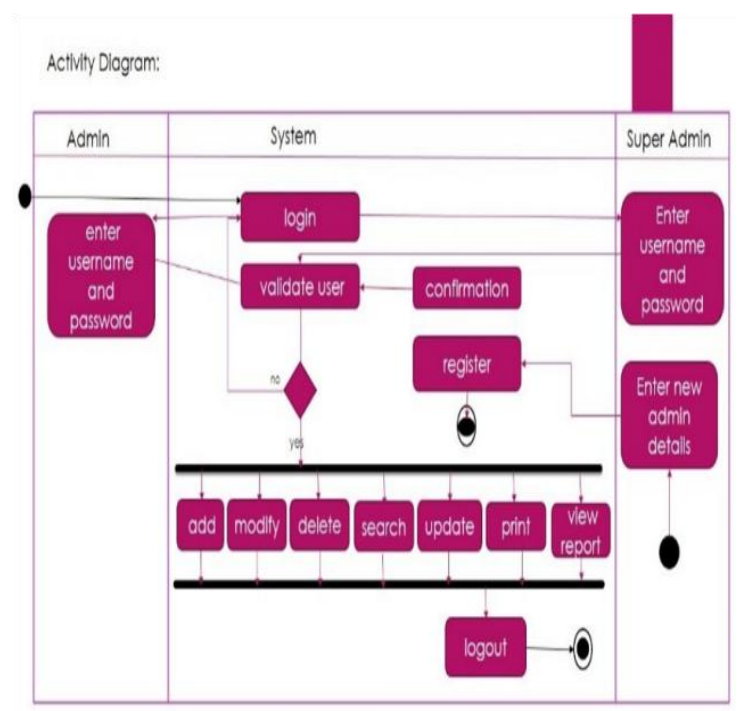

Fig. 1 : Activity Diagram

\section{ACKNOWLEDGEMENT}

An Endeavour over a period of time can be successful only with the constant support and guidance from our well-wishers, hence we take this opportunity to express our gratitude to all those who encouraged us in getting a head start on our project work.

We are immensely grateful to our Principal, for providing the needed facilities creating a comfortable environment required for the project.

We also thank Mrs. Manjusha Sanke, Head of the Department of Information Technology who assured us every support from the institute.

Our sincere appreciation to our Internal guide, Ms. Melancy Mascarenhas, Assistant Professor, IT Department and Mr. Rajendra Satarkar deputy Manager IT, CIPLA for providing us with excellent guidance, direction and constant support in organization, planning and scheduling the project phases. 
We would like to thank Mrs. Prajakta Tanksali, project coordinator, IT Department, for her support in organization, planning and scheduling the project phases.

Besides we are also grateful to our friends and well-wishers who provided us with lots of support.

Lastly, we are grateful to whole of the Information Technology staff for providing us with the lab facilities and co-operation for our project.

\section{REFERENCES}

[1] S. Angel Raphella, S. Gomathi Nathan and G. Chitra "Inventory Management-A Case Study", International Journal of Emerging Research in Management and
Technology, ISSN: 2278-9359, vol.3 (3) June 2014, pp. 94-102.

[2] P.G. Matsebatlela and K.Mpofu,'Inventory Management Framework to Minimize Supply and demand Mismatch on a Manufacturing Organization", International Federation of Automatic Control, vol.3, no.48, Mar 2015, pp-260-265

[3] Harry, E. G. "Tracking inventory", London, underwood pitman, Haward University, March- April, pp.64-73

[4] Sande, O.F. (2013). "Automated Warehouse Management System". Journal of Toronto. Vol.1, pp.4551 